\title{
Uniform observability estimates for the 1-D discretized wave equation and the random choice method
}

\author{
Jean-Michel Coron ${ }^{\mathrm{a}, \mathrm{b}, \mathrm{c}, 1}$, Sylvain Ervedoza ${ }^{\mathrm{d}, 1}$ Olivier Glass ${ }^{\mathrm{a}, \mathrm{b}, 1}$ \\ ${ }^{\text {a } U P M C ~ U n i v ~ P a r i s ~ 06, ~ U M R ~ 7598, ~ L a b o r a t o i r e ~ J a c q u e s-L o u i s ~ L i o n s, ~ 4 ~ p l a c e ~ J u s s i e u, ~ F-75005, ~ P a r i s, ~ F r a n c e ~}$ \\ ${ }^{\mathrm{b}}$ CNRS, UMR 7598, Laboratoire Jacques-Louis Lions, F-75005, Paris, France \\ ${ }^{\mathrm{c}}$ Institut Universitaire de France \\ ${ }^{\mathrm{d}}$ Laboratoire de Mathématiques de Versailles, Université de Versailles Saint Quentin en Yvelines, 45 avenue des \\ États-Unis, 78035 Versailles, France \\ Received $* * * * * ;$ accepted after revision +++++ \\ Presented by
}

\begin{abstract}
In this Note, we consider the 1-dimensional wave equation, discretized by means of Glimm's random choice method. We prove that for almost every choice of the random parameter, the observability estimate is true asymptotically, uniformly with respect to the discretization parameters. To cite this article: A. Name1, A. Name2, C. R. Acad. Sci. Paris, Ser. I 340 (2005).

\section{Résumé}

Estimées d'observabilité uniformes pour l'équation des ondes unidimensionnelle et méthode du choix aléatoire Dans cette Note, nous considérons l'équation des ondes unidimensionnelle discrétisée selon la méthode du choix aléatoire due à J. Glimm. Nous établissons que pour presque tout choix de la variable aléatoire, l'estimée d'observabilité pour cette équation est vraie asymptotiquement, uniformément en les paramètres de discrétisation. Pour citer cet article: A. Name1, A. Name2, C. R. Acad. Sci. Paris, Ser. I 340 (2005).
\end{abstract}

\section{Version française abrégée}

Soit $c>0$. On considère en dimension un d'espace l'équation de transport à vitesse constante (1) et l'équation des ondes (2), où pour simplifier le problème nous avons posé les équations dans le cercle $\mathbb{T}:=\mathbb{R} / \mathbb{Z}$. On remplacera (2) par le système (3) où l'on a utilisé les notations (4).

\footnotetext{
1 The authors are partially supported by the ANR, projects C-QUID and ContrôleFlux.

Email addresses: coron@ann.jussieu.fr (Jean-Michel Coron), sylvain.ervedoza@math.uvsq.fr (Sylvain Ervedoza), glass@ann.jussieu.fr (Olivier Glass).
} 
Ces deux équations sont des cas très particuliers de systèmes de lois de conservation unidimensionnels pour lesquelles J. Glimm a obtenu un résultat très général d'existence de solutions au moyen d'un algorithme d'approximation de solutions utilisant une suite de variables aléatoires, connu désormais sous le nom de méthode du choix aléatoire ou schéma de Glimm [1]. Le but de cette Note est de considérer l'inégalité classique d'observabilité (5) pour ces équations, lorsque celles-ci sont discrétisées au moyen de cet algorithme. La problématique d'obtenir de telles estimées uniformément en les paramètres de discrétisation est un sujet en pleine expansion, voir en particulier [2,5]. Même pour les équations (1) et (2), dans le cadre d'une discrétisation par différences finies ou éléments finis, le schéma numérique engendre des rayons parasites haute fréquence qui se propagent à vitesse nulle, conduisant à une observabilité non uniforme par rapport aux paramètres de discrétisation (voir l'appendice pour l'équation (1)). Nous allons voir qu'au contraire dans le cadre de la méthode du choix aléatoire, avec une très bonne probabilité, on obtient une inégalité d'observabilité uniforme par rapport aux paramètres de discrétisation.

Rappelons brièvement comment sont construites les approximations dans le cadre de la méthode du choix aléatoire (voir la Figure 1).

Soit $\left(\theta_{i}\right)_{i \in \mathbb{N}} \in[-1,1]^{\mathbb{N}}$ une suite de tirages indépendants uniformes sur $[-1,1]$; l'ensemble $[-1,1]^{\mathbb{N}}$ est bien entendu muni de sa tribu et de sa mesure de probabilité $\mathbb{P}$ habituelle. On choisira des pas de discrétisation $\Delta t$ en temps et $\Delta x$ en position, respectant la condition de Courant-Friedrich-Lewy (6). Ils seront en outre choisis selon un facteur d'échelle hyperbolique, au sens où, pour une certaine constante $M>0$, l'inégalité (7) est satisfaite.

Le domaine spatial est divisé en intervalles $[k \Delta x,(k+1) \Delta x$ [ et au temps $t=n \Delta t$ fixé, l'approximation est constante sur les mailles $[k \Delta x,(k+2) \Delta x[$ avec $k+n \equiv 0(\bmod 2)$. La donnée initiale $u(0, x)$ est approchée dans $L^{2}$ par une fonction $u_{0}$ constante dans les mailles [ $2 k \Delta x,(2 k+2) \Delta x[$, obtenue par exemple par intégration de la donnée initiale dans chaque maille. Le passage de l'étape $n$ à l'étape $n+1$ s'opère de la manière suivante : à chaque discontinuité $k \Delta x$ de l'approximation au temps $n \Delta t$ avec $k+n \equiv 0(\bmod 2)$, on résout le problème de Riemann associé à la discontinuité entre la valeur dans la maille $[(k-2) \Delta x, k \Delta x[$ et celle dans la maille $[k \Delta x,(k+2) \Delta x[$; soit $R(t, x)$ la solution ainsi déterminée. La valeur choisie dans la maille suivante $[(k-1) \Delta x,(k+1) \Delta x$ [ est alors donnée par (8). Autrement dit, la donnée dans la maille suivante est choisie « au hasard » dans la solution du problème de Riemann (au moyen d'une variable aléatoire $\theta_{n}$ ne dépendant que du temps $n \Delta t$ ). Le schéma est représenté dans la Figure 1 ; la place du choix aléatoire y est désignée par un point noir. Étant donnés une donnée initiale $u_{0}$, deux pas de discrétisation $\Delta t$ et $\Delta x$ satisfaisant (6) et $\left(\theta_{i}\right)_{i \in \mathbb{N}} \in[-1,1]^{\mathbb{N}}$, on notera $u\left[u_{0}, \Delta t, \Delta x,\left(\theta_{i}\right)\right]$ l'approximation obtenue par le procédé précédent, et $u_{n}^{k}\left[u_{0}, \Delta t, \Delta x,\left(\theta_{i}\right)\right]:=u\left[u_{0}, \Delta t, \Delta x,\left(\theta_{i}\right)\right](n \Delta t, k \Delta x)$.

Le but de cette Note est de prouver le résultat suivant.

Théorème 0.1 Étant donnés un temps $T>1 / c, \Delta t$ et $\Delta x$ satisfaisant (6) et $N:=T / \Delta t \in 2 \mathbb{N}, K:=$ $1 / \Delta x \in 2 \mathbb{N}$, il existe un ensemble $\Theta(\Delta t, \Delta x) \subset[-1,1]^{\mathbb{N}}$ de choix aléatoires $\left(\theta_{i}\right)_{i \in \mathbb{N}}$ tel que pour toute donnée initiale $u_{0}$ discrète, l'inégalité d'observabilité (9) en $x=0$ pour les approximations par méthode du choix aléatoire des solutions de (1) et (3) est satisfaite et de plus $\Theta(\Delta t, \Delta x)$ satisfait (10).

Corollaire 0.2 Soit $M>c$ et $T>1 / c$. Il existe un ensemble $\Theta$ de choix aléatoires de mesure pleine tel que pour tout $\left(\theta_{i}\right)_{i \in \mathbb{N}} \in \Theta$, il existe $\varepsilon>0$ tel que pour tout $\Delta t, \Delta x>0$ satisfaisant $T / \Delta t \in 2 \mathbb{N}$, $1 / \Delta x \in 2 \mathbb{N}$, (6), (7) et $\Delta t \leq \varepsilon$ et toute donnée initiale, l'inégalité (9) est satisfaite.

\section{Introduction}

Let $c>0$. We consider in one space dimension the transport equation with constant speed $c$

$$
u_{t}+c u_{x}=0 \text { for }(t, x) \in[0, T] \times \mathbb{T},
$$

and the wave equation 


$$
v_{t t}-c^{2} v_{x x}=0 \text { for }(t, x) \in[0, T] \times \mathbb{T},
$$

where for simplicity we have posed the problem on the circle $\mathbb{T}:=\mathbb{R} / \mathbb{Z}$. We replace (2) by the equivalent system

$$
u_{+, t}+c u_{+, x}=0 \text { and } u_{-, t}-c u_{-, x}=0 \text { for }(t, x) \in[0, T] \times \mathbb{T}
$$

where

$$
u_{+}:=v_{t}-c v_{x}, u_{-}:=v_{t}+c v_{x} \text { and } u:=\left(u_{-}, u_{+}\right) .
$$

These two equations are very particular cases of one-dimensional hyperbolic systems of conservation laws for which J. Glimm obtained a very general result for the existence of solutions, by means of a particular numerical scheme using a random sequence, known as the random choice method or Glimm's scheme [1]. The purpose of this Note is to consider the classical observability inequalities for these equations for $T \geq 1 / c$ :

$$
\left\|u_{\mid t=0}\right\|_{L^{2}(\mathbb{T})}^{2} \leq c\left\|u_{\mid x=0}\right\|_{L^{2}(0, T)}^{2} \text { for Eq. (1) and }\left\|u_{\mid t=0}\right\|_{L^{2}\left(\mathbb{T} ; \mathbb{R}^{2}\right)}^{2} \leq c\left\|u_{\mid x=0}\right\|_{L^{2}\left((0, T) ; \mathbb{R}^{2}\right)}^{2} \text { for Eq. (3), }
$$

when they are discretized by means of this algorithm. Note that both $T \geq 1 / c$ and the constants in (5) are optimal. Actually we could treat by the same approach the slightly more technical case of internal observability inequalities: for $a \in(0,1)$ and for every $T>1-a$, one has

$$
\left\|u_{\mid t=0}\right\|_{L^{2}(\mathbb{T})} \lesssim\|u\|_{L^{2}((0, T) \times(0, a))} \text { for Eq. (1) and }\left\|u_{\mid t=0}\right\|_{L^{2}\left(\mathbb{T} ; \mathbb{R}^{2}\right)} \lesssim\|u\|_{L^{2}\left((0, T) \times(0, a) ; \mathbb{R}^{2}\right)} \text { for Eq. (3), }
$$

see for instance $[4$, Chapitre VII, Lemme 2.3, p. 411] in the case of the wave equation.

This issue to get uniform observability estimates with respect to the discretization parameters has been extensively studied in recent years, see in particular [2,5]. Even for the simple equations (1) and (2), in the context of finite differences and finite elements methods, the numerical scheme generates high frequency spurious waves which propagate at zero speed, making the observability inequality non-uniform with respect to the discretization parameters (see the Appendix for Equation (1)). We will prove that on the contrary in the framework of the random choice method, with a very good probability, we obtain an observability inequality which is uniform with respect to the discretization parameters.

Let us briefly recall how the approximations of the random choice method are constructed.

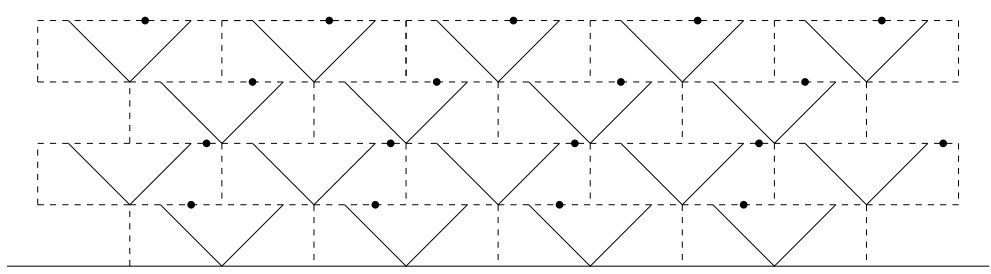

Figure 1. Glimm's scheme for the wave equation

Let $\left(\theta_{i}\right)_{i \in \mathbb{N}} \in[-1,1]^{\mathbb{N}}$ a sequence of independent random variables uniformly distributed on $[-1,1] ;$ the set $[-1,1]^{\mathbb{N}}$ is endowed with its product $\sigma$-algebra and usual probability measure $\mathbb{P}$. The discretization parameters, $\Delta t$ in time and $\Delta x$ in space, will satisfy the Courant-Friedrich-Lewy condition:

$$
\frac{\Delta x}{\Delta t}>c .
$$

They will also be chosen in a hyperbolic scaling, in the sense that for some constant $M>0$

$$
\frac{\Delta x}{\Delta t} \leq M
$$

The space domain is divided into intervals $[k \Delta x,(k+1) \Delta x)$ and at fixed time $t=n \Delta t$, the approximation is constant on the intervals $[k \Delta x,(k+2) \Delta x)$ with $k+n \equiv 0(\bmod 2)$. The initial data $u(0, x)$ is 
approximated in $L^{2}$ by a function $u_{0}$ which is constant in each interval $[2 k \Delta x,(2 k+2) \Delta x)$, for instance obtained by means of integration in this interval.

The passage from the step $n$ to the step $n+1$ is done in the following way: for each discontinuity $k \Delta x$ of the approximation at time $n \Delta t$ with $k+n \equiv 0(\bmod 2)$, one solves the Riemann problem corresponding to the discontinuity between the value in the interval $[(k-2) \Delta x, k \Delta x)$ and the one in the interval $[k \Delta x,(k+2) \Delta x)$. Let $R(t, x)$ the solution of this Riemann problem. The value chosen in the next interval $[(k-1) \Delta x,(k+1) \Delta x)$ is the following one:

$$
u((n+1) \Delta t, x)=R\left((n+1) \Delta t, k \Delta x+\theta_{n+1} \Delta x\right) \forall x \in[(k-1) \Delta x,(k+1) \Delta x) .
$$

In other words, the data in the next interval is randomly chosen inside the solution of the Riemann problem (by means of a random variable $\theta_{n}$ depending on the time $n \Delta t$ only). We have represented the scheme in Figure 1; the spot of the random choice is represented by a black dot. Given an initial data $u_{0}$, two discretization parameters $\Delta t$ and $\Delta x$ fulfilling $(6)$ and $\left(\theta_{i}\right)_{i \in \mathbb{N}} \in[-1,1]^{\mathbb{N}}$, we denote by $u\left[u_{0}, \Delta t, \Delta x,\left(\theta_{i}\right)\right]$ the approximation obtained through the above numerical algorithm, and by $u_{n}^{k}\left[u_{0}, \Delta t, \Delta x,\left(\theta_{i}\right)\right]:=u\left[u_{0}, \Delta t, \Delta x,\left(\theta_{i}\right)\right](n \Delta t, k \Delta x)$.

The purpose of this Note is to establish the following result.

Theorem 1.1 Given a time $T>1 / c, \Delta t$ and $\Delta x$ satisfying (6) and $N:=T / \Delta t \in 2 \mathbb{N}, K:=1 / \Delta x \in 2 \mathbb{N}$, there exists a set $\Theta(\Delta t, \Delta x) \subset[-1,1]^{\mathbb{N}}$ of random choices $\left(\theta_{i}\right)_{i \in \mathbb{N}}$ such that for any discrete initial data $u_{0}$, the observability inequality at $x=0$ for Glimm's approximations of the solutions of (1) and (3)

$$
\sum_{j=0}^{(K / 2)-1}\left|u_{0}^{2 j+1}\left[u_{0}, \Delta t, \Delta x,\left(\theta_{i}\right)\right]\right|^{2} \leq \sum_{l=0}^{(N / 2)-1}\left|u_{2 l+1}^{0}\left[u_{0}, \Delta t, \Delta x,\left(\theta_{i}\right)\right]\right|^{2},
$$

is satisfied and moreover

$$
\mathbb{P}\left({ }^{c} \Theta(\Delta t, \Delta x)\right) \leq(1+\delta) \exp \left(-\frac{T}{2 \Delta t}\left(\frac{\Delta t}{\Delta x}\right)^{2}\left(\frac{1}{T}-c\right)^{2}\right),
$$

with $\delta=0$ for the transport equation and $\delta=1$ for the wave equation.

Corollary 1.2 Let $M>c$ and $T>1 / c$. There exists a set $\Theta$ of random choices, which has full measure, such that for any $\left(\theta_{i}\right)_{i \in \mathbb{N}} \in \Theta$, there exists $\varepsilon>0$ such that for any $\Delta t, \Delta x>0$ satisfying $T / \Delta t \in 2 \mathbb{N}$, $1 / \Delta x \in 2 \mathbb{N}$, (6), (7) and $\Delta t \leq \varepsilon$ and any initial data, the inequality (9) is satisfied.

\section{Proofs of Theorem 1.1 and Corollary 1.2}

Proof of Theorem 1.1. Let us treat the case of equation (1). The main idea is to interpret the scheme in terms of a random walk.

We begin by describing the solution of the Riemann problem. Given the states $u_{l}$ in the left interval $[(k-2) \Delta x, k \Delta x)$ and $u_{r}$ in the right interval $[k \Delta x,(k+2) \Delta x)$ at the time $n \Delta t$, the solution $R$ of the Riemann problem in the interval $[(k-1) \Delta x,(k+1) \Delta x)$ at the time $(n+1) \Delta t$ is given by

$$
R((n+1) \Delta t, x)=u_{l} \text { in }[(k-1) \Delta x, k \Delta x+c \Delta t] \text { and } R((n+1) \Delta t, x)=u_{r} \text { in }[k \Delta x+c \Delta t,(k+1) \Delta x) .
$$

It then follows from (8) that

$$
u_{n+1}^{k}=u_{n}^{k-1} \text { if } \theta_{n}<c \frac{\Delta t}{\Delta x} \text { and } u_{n+1}^{k}=u_{n}^{k+1} \text { if } \theta_{n}>c \frac{\Delta t}{\Delta x} .
$$

Hence the values of $u_{n}^{k}$ are propagated along the trajectories of a (non-symmetric) random walk with probability $p_{l}:=\frac{1}{2}-c \frac{\Delta t}{2 \Delta x}$ of moving to the left and $p_{r}:=\frac{1}{2}+c \frac{\Delta t}{2 \Delta x}$ of moving to the right. Let

$$
X_{i}:=\mathbf{I}_{\theta_{i}<c \frac{\Delta t}{\Delta x}}-\mathbf{I}_{\theta_{i}>c \frac{\Delta t}{\Delta x}},
$$


where by $\mathbf{I}_{A}$ we denote the indicator function of the event $A$. Then $\left(X_{i}\right)_{i \in \mathbb{N}}$ is a sequence of independent identically distributed variables with $X_{i}=-1$ with probability $p_{l}$ and $X_{i}=1$ with probability $p_{r}$. Let $S_{n}=X_{1}+\cdots+X_{n}$. It is a consequence of (12) and (13) that the following characteristics formula holds

$$
u_{n}^{k}=u_{0}^{k^{\prime}} \text { with } k^{\prime}:=k-S_{n}(\bmod K) .
$$

Now a sufficient condition for inequality (9) to hold is that $S_{N} \geq K$, because under this condition for each $j \in\left\{0, \ldots, \frac{K}{2}-1\right\}$, there is some $l \in\left\{0, \ldots, \frac{N}{2}-1\right\}$ such that the point $S_{2 l+1}+2 j+1=K$. Roughly speaking, each point $x=(2 j+1) \Delta x$ has been driven at some time through $x=1$ by the shift $S_{2 l+1} \Delta x$. Call $\Theta(\Delta t, \Delta x)$ the set of $\left(\theta_{i}\right)_{i \in \mathbb{N}}$ for which $S_{N} \geq K$. Now, Hoeffding's inequality [3, p. 448] reads

$$
\forall n \in \mathbb{N}, \quad \mathbb{P}\left(S_{n}-\mathbb{E}\left(S_{n}\right) \leq-n \alpha\right) \leq \exp \left(-\frac{n \alpha^{2}}{2}\right) .
$$

Taking $n=N=T / \Delta t$, we get $\mathbb{E}\left(S_{n}\right)=\mathbb{E}\left(S_{N}\right)=c N \frac{\Delta t}{\Delta x}=K+\frac{c T-1}{\Delta x}$. Choosing $\alpha=\frac{c T-1}{N \Delta x}$, we infer that

$$
\mathbb{P}\left({ }^{c} \Theta(\Delta t, \Delta x)\right) \leq \exp \left(-\frac{\Delta t}{2 T(\Delta x)^{2}}(c T-1)^{2}\right),
$$

which gives (9).

For what concerns the wave equation, we use the decoupled form (3); then (12) has to be replaced by

$$
u_{+, n+1}^{k}=\left\{\begin{array}{l}
u_{+, n}^{k-1} \text { when } \theta_{n}<c \frac{\Delta t}{\Delta x}, \\
u_{+, n}^{k+1} \text { when } \theta_{n}>c \frac{\Delta t}{\Delta x},
\end{array} \text { and } u_{-, n+1}^{k}=\left\{\begin{array}{l}
u_{-, n}^{k-1} \text { when } \theta_{n}<-c \frac{\Delta t}{\Delta x}, \\
u_{-, n}^{k+1} \text { when } \theta_{n}>-c \frac{\Delta t}{\Delta x} .
\end{array}\right.\right.
$$

This yields two formulas (14) for $u_{+}$and $u_{-}$for two different (but correlated) random walks, hence the $1+\delta$ in formula (9).

Proof of Corollary 1.2. Introduce the countable set

$$
D_{\varepsilon}:=\left\{(\Delta t, \Delta x) \in(0,1)^{2} / \Delta t<\varepsilon, c<\frac{\Delta x}{\Delta t} \leq M \text { and } T / \Delta t \in 2 \mathbb{N}, 1 / \Delta x \in 2 \mathbb{N}\right\} .
$$

Given $\Delta t>0$, we denote $\tilde{D}_{\varepsilon}(\Delta t):=\left\{\Delta x /(\Delta t, \Delta x) \in D_{\varepsilon}\right\}$. Note that due to (6) and (7), we have

$$
\operatorname{Card}\left(\tilde{D}_{\varepsilon}(\Delta t)\right) \leq \frac{1}{2}\left(\frac{1}{c}-\frac{1}{M}\right) \frac{1}{\Delta t}+1
$$

We introduce $\Theta$ in the following way

$$
\Theta:=\bigcup_{\varepsilon>0} \bigcap_{(\Delta t, \Delta x) \in D_{\varepsilon}} \Theta(\Delta t, \Delta x)
$$

Let us show that $\mathbb{P}\left({ }^{c} \Theta\right)=0$. We have

$$
\begin{aligned}
\mathbb{P}\left(\bigcup_{(\Delta t, \Delta x) \in D_{\varepsilon}}{ }^{c} \Theta(\Delta t, \Delta x)\right) & \leq \sum_{D_{\varepsilon}} \exp \left(-\frac{1}{2 T M^{2}} \frac{(c T-1)^{2}}{\Delta t}\right) \\
& \leq \sum_{\substack{\Delta t \in T / 2 \mathbb{N} \\
\Delta t<\varepsilon}} \sum_{\substack{\Delta x \in \tilde{D}_{\varepsilon}(\Delta t)\\
}} \exp \left(-\frac{1}{2 T M^{2}} \frac{(c T-1)^{2}}{\Delta t}\right) \\
& \leq \sum_{\substack{\Delta t \in T / 2 \mathbb{N} \\
\Delta t<\varepsilon}}\left(\frac{1}{2}\left(\frac{1}{c}-\frac{1}{M}\right) \frac{1}{\Delta t}+1\right) \exp \left(-\frac{1}{2 T M^{2}} \frac{(c T-1)^{2}}{\Delta t}\right)
\end{aligned}
$$

It is clear that the latter is finite and converges towards 0 as $\varepsilon \rightarrow 0^{+}$. Since $\cup_{(\Delta t, \Delta x) \in D_{\varepsilon}}{ }^{c} \Theta(\Delta t, \Delta x)$ is non-decreasing as a function of $\varepsilon$, we infer $\mathbb{P}\left({ }^{c} \Theta\right)=0$. This concludes the proof of Corollary 1.2. 
Remark 1 We first point out that our method leads to the optimal time for observability. For what concerns the constant in the observability inequality, we rewrite (9) as follows

$$
2 \Delta x \sum_{j=0}^{(K / 2)-1}\left|u_{0}^{2 j+1}\left[u_{0}, \Delta t, \Delta x,\left(\theta_{i}\right)\right]\right|^{2} \leq\left(\frac{\Delta x}{\Delta t}\right) 2 \Delta t \sum_{l=0}^{(N / 2)-1}\left|u_{2 l+1}^{0}\left[u_{0}, \Delta t, \Delta x,\left(\theta_{i}\right)\right]\right|^{2},
$$

which yields $M$ as an observability constant. Note that by (6) and (7), one can let $M \rightarrow c^{+}$and therefore get an almost optimal observability inequality, uniformly with respect to the discretization parameters.

\section{Appendix A. Lack of observability property for the transport equation (1).}

For completeness, we briefly present the proof of the lack of uniform observability properties for the following natural finite difference space semi-discrete approximations of the transport equation (1):

$$
\partial_{t} u^{j}+c \frac{u^{j+1}-u^{j-1}}{2 \Delta x}=0 \text { for }(t, j) \in[0, T] \times\{0, \cdots, K-1\}, \text { and } u^{j}(0)=u_{0}^{j} \text { and } u^{K}=u^{0} .
$$

Here, the notations are the standard ones and $u^{j}(t)$ is an approximation of $u(j \Delta x, t)$.

Let us check that, whatever $T>0$ is,

$$
\lim _{\Delta x \rightarrow 0^{+}} \inf _{u_{0}}\left\{\int_{0}^{T}\left|u^{0}(t)\right|^{2} d t / \Delta x \sum_{j=0}^{K-1}\left|u_{0}^{j}\right|^{2}\right\}=0
$$

This can be proved by considering the solution

$$
u^{j}(t)=\exp \left(i c t \frac{\sin (k \pi \Delta x)}{\Delta x}\right) \exp (i k \pi j \Delta x)-\exp \left(i c t \frac{\sin ((k+1) \pi \Delta x)}{\Delta x}\right) \exp (i(k+1) \pi j \Delta x),
$$

with $k=\lfloor K / 2\rfloor$. This is a solution of (A.1) for which explicit computations yield $\Delta x \sum_{j=0}^{K-1}\left|u_{0}^{j}\right|^{2}=2$ and

$$
\begin{aligned}
\int_{0}^{T}\left|u^{0}(t)\right|^{2} d t & =4 \int_{0}^{T} \sin ^{2}\left(\frac{c t}{\Delta x} \sin \left(\frac{\pi \Delta x}{2}\right) \cos ((k+1 / 2) \pi \Delta x)\right) d t \\
& \leq \int_{0}^{T} \pi^{2} c^{2} t^{2} \cos ^{2}((k+1 / 2) \pi \Delta x) d t \leq \frac{\pi^{2} c^{2} T^{3}}{3} \cos ^{2}((k+1 / 2) \pi \Delta x) .
\end{aligned}
$$

This last term goes to zero as $\Delta x \rightarrow 0$ for $k=\lfloor K / 2\rfloor$ since $(k+1 / 2) \Delta x \simeq 1 / 2$.

\section{References}

[1] Glimm J., Solutions in the large for nonlinear hyperbolic systems of equations. Comm. Pure Appl. Math. 18 (1965), pp. 697-715.

[2] Glowinski R., Lions J.-L., He J., Exact and approximate controllability for distributed parameter systems. A numerical approach. Encyclopedia of Mathematics and its Applications, 117. Cambridge University Press, Cambridge, 2008.

[3] Grimmett G. R., Stirzaker D. R., Probability and random processes. Second edition. The Clarendon Press, Oxford University Press, New York, 1992.

[4] Lions J.-L., Contrôlabilité exacte, stabilisation et perturbations de systèmes distribués, Tomes 1 \& 2. Masson, RMA 8 \& 9, Paris 1988.

[5] Zuazua E., Propagation, observation, and control of waves approximated by finite difference methods, SIAM Rev. 47 (2005), no. 2, 197-243 